\title{
Star-Supermagic Decompositions of the Complete Bipartite Graph Minus a One-Factor
}

\author{
Tanawat Wichianpaisarn $^{1}$ and Uthoomporn Mato ${ }^{2}$ \\ ${ }^{1}$ Department of Mathematics, Faculty of Applied Science, King Mongkut's University of Technology North Bangkok, \\ Pracharat 1 Rd., Bangkok 10800, Thailand \\ ${ }^{2}$ Department of Mathematics, Faculty of Science, Srinakharinwirot University, Bangkok 10110, Thailand
}

Correspondence should be addressed to Tanawat Wichianpaisarn; tanawat.w@sci.kmutnb.ac.th

Received 23 March 2017; Accepted 30 May 2017; Published 21 June 2017

Academic Editor: Dalibor Froncek

Copyright (C) 2017 Tanawat Wichianpaisarn and Uthoomporn Mato. This is an open access article distributed under the Creative Commons Attribution License, which permits unrestricted use, distribution, and reproduction in any medium, provided the original work is properly cited.

Let $G$ be a graph and let $H$ be a subgraph of $G$. Assume that $G$ has an $H$-decomposition $T=\left\{H_{1}, H_{2}, \ldots, H_{t}\right\}$ such that $H_{i} \cong H$ for all $1 \leq i \leq t$. An $H$-supermagic decomposition of $G$ is a bijection $f: V(G) \cup E(G) \rightarrow\{1,2, \ldots,|V(G)|+|E(G)|\}$ such that $\sum_{v \in V\left(H_{i}\right)} f(v)+\sum_{e \in E\left(H_{i}\right)} f(e)$ is a constant $k$ for each $H_{i}$ in the decomposition $T$ and $f(V(G))=\{1,2, \ldots,|V(G)|\}$. If $G$ admits an $H$ supermagic decomposition, then $G$ is called $H$-supermagic decomposable. In this paper, we give necessary and sufficient conditions for the existence of $K_{1, n-1}$-supermagic decomposition of the complete bipartite graph $K_{n, n}$ minus a one-factor.

\section{Introduction}

All graphs in this paper are finite and simple. We use terminologies of graphs from West's textbook [1]. If $m$ and $n$ are integers with $m \leq n$, we denote $\{m, m+1, \ldots, n\}$ by $[m, n]$.

Let $G$ be a graph. A covering of $G$ is a family of subgraphs $H_{1}, H_{2}, \ldots, H_{t}$ such that each edge of $G$ belongs to at least one of the subgraphs $H_{i}$. If $H_{i} \cong H$, for all $i \in[1, t]$, then the covering is called an $H$-covering of $G$.

Assume that $G$ has an $H$-covering. An $H$-magic labeling of $G$ is a bijection $f: V(G) \cup E(G) \rightarrow[1,|V(G)|+|E(G)|]$ such that $\sum_{v \in V\left(H_{i}\right)} f(v)+\sum_{e \in E\left(H_{i}\right)} f(e)$ is a constant $k$ for each $H_{i}$ in the $H$-covering, and $k$ is called a magic constant. An $H$-magic labeling $f$ is called an $H$-supermagic labeling if $f(V(G))=$ $[1,|V(G)|]$. A graph $G$ is said to be $H$-supermagic if $G$ admits an $H$-magic labeling.

The $H$-supermagic labeling was first introduced by Gutiérrez and Lladó [2] in 2005. They proved that many classes of graphs are $H$-supermagic, such as the complete bipartite graphs $K_{m, n}$ and the star $K_{1, n}$ are $K_{1, l}$-supermagic for some positive integer $l$. In 2007, Lladó and Moragas [3] studied some windmills, wheels, and thetas which are
$H$-supermagic. For example, they showed that the wheel $W_{n}$ for odd $n$ is $C_{3}$-supermagic.

In 2010, Ngurah et al. [4] proved that the fan $P_{n} \vee K_{1}$, the triangle ladder $T L_{n}$, and $K_{1, n} \vee K_{1}$ are $C_{3}$-supermagic. Furthermore, they showed that the book $B_{n}$, the ladder $L_{n}$, and the grid $P_{m} \times P_{n}$ are $C_{4}$-supermagic.

An $H$-decomposition of a graph $G$ is an $H$-covering of $G$ which forms a partition of the edge set of $G$. If $G$ has an $H$-decomposition, then $G$ is called $H$-decomposable. Assume that $G$ is $H$-decomposable. If $G$ admits an $H$-supermagic labeling, then $G$ is said to be $H$-supermagic decomposable or $G$ has an $H$-supermagic decomposition.

The concept of an $H$-supermagic decomposition of $G$ arises from the combination between graph labelings and graph decompositions which was introduced by Liang [5] in 2012. He found the conditions for the existence of $C_{2 k}$ supermagic decomposition of the complete $n$-partite graph as well as of multiple copies of it. Moreover, in 2015, Marimuthu and Kumar [6] showed that the complete bipartite graph $K_{n, n}$ is $K_{1, n}$-supermagic decomposable. For more information about graph labelings, please see Gallian's survey [7]. 
Motivated by the results of these authors, we are going to find necessary and sufficient conditions for the existence of an $H$-supermagic decomposition of the complete bipartite graph $K_{n, n}$ minus a one-factor, where $H \cong K_{1, n-1}$. Hereafter, if $H \cong$ $K_{1, n}$, for some positive integer $n$, then $H$ is called an $n$-star subgraph. The notation of an $n$-star-subgraph was introduced by Akiyama and Kano [8].

\section{Main Result}

In this section, we consider the graph $K_{n, n}-I$, where $I$ is a one-factor of $K_{n, n}$ and $n \geq 2$. Note that $K_{n, n}-I$ is $(n-1)$ star-decomposable for all $n \geq 2$. Since $\left|V\left(K_{n, n}-I\right)\right|=2 n$ and $\left|E\left(K_{n, n}-I\right)\right|=n^{2}-n$, we have $\left|V\left(K_{n, n}-I\right)\right|+\left|E\left(K_{n, n}-I\right)\right|=$ $n^{2}+n$

Theorem 1. Let $n$ be a positive integer with $n \equiv 2(\bmod 4)$. Then $K_{n, n}-I$ is not an $(n-1)$-star-supermagic decomposable graph.

Proof. Suppose that $K_{n, n}-I$ is $(n-1)$-star-supermagic decomposable. Let $f$ be an $(n-1)$-star-supermagic labeling of $K_{n, n}-I$ with the magic constant $k$. Let $U=\left\{u_{1}, u_{2}, \ldots, u_{n}\right\}$ and $V=\left\{v_{1}, v_{2}, \ldots, v_{n}\right\}$ be vertex-bipartitions of $K_{n, n}-I$. Let $\left\{H_{1}, H_{2}, \ldots, H_{n}\right\}$ be an $(n-1)$-star-decomposition of $K_{n, n}-I$, where $H_{i}$ contains one vertex of $U$ and $n-1$ vertices of $V$ for all $i \in[1,2 n]$.

Consider the labeling $f$; assume that all vertices of $U$ are labeled by $\left\{a_{1}, a_{2}, \ldots, a_{n}\right\}$ and all vertices of $V$ are labeled by $\left\{a_{n+1}, a_{n+2}, \ldots, a_{2 n}\right\}$. Note that $a_{i} \in[1,2 n]$ for all $i \in[1,2 n]$. Then

$$
\begin{aligned}
& \sum_{i=1}^{n} \sum_{v \in V\left(H_{i}\right)} f(v)+\sum_{i=1}^{n} \sum_{e \in E\left(H_{i}\right)} f(e)=\left[\left(a_{1}+a_{2}+\cdots+a_{n}\right)\right. \\
& \left.+(n-1)\left(a_{n+1}+a_{n+2}+\cdots+a_{2 n}\right)\right]+[(2 n+1) \\
& \left.+(2 n+2)+\cdots+\left(n^{2}+n\right)\right]=\left[\left(a_{1}+a_{2}+\cdots+a_{2 n}\right)\right. \\
& \left.+(n-2)\left(a_{n+1}+a_{n+2}+\cdots+a_{2 n}\right)\right]+\sum_{i=2 n+1}^{n^{2}+n} i=\sum_{i=1}^{2 n} i \\
& +(n-2)\left(a_{n+1}+a_{n+2}+\cdots+a_{2 n}\right)+\sum_{i=2 n+1}^{n^{2}+n} i=\sum_{i=1}^{n^{2}+n} i \\
& +(n-2)\left(a_{n+1}+a_{n+2}+\cdots+a_{2 n}\right) \\
& +\frac{\left(n^{2}+n\right)\left(n^{2}+n+1\right)}{2}+(n-2)\left(a_{n+1}+a_{n+2}+\cdots\right. \\
& \left.+a_{2 n}\right) .
\end{aligned}
$$

Since $n \equiv 2(\bmod 4)$, we have $2 \mid n$ but $4+n$; that is, $n=2(2 k+1)$ for some integer $k$. Note that $\left(n^{2}+n\right)\left(n^{2}+\right.$ $n+1) / 2$ is odd and $(n-2)\left(a_{n+1}+a_{n+2}+\cdots+a_{2 n}\right)$ is even. Thus, $\sum_{i=1}^{n} \sum_{v \in V\left(H_{i}\right)} f(v)+\sum_{i=1}^{n} \sum_{e \in E\left(H_{i}\right)} f(e)$ is odd. We know that $\sum_{i=1}^{n} \sum_{v \in V\left(H_{i}\right)} f(v)+\sum_{i=1}^{n} \sum_{e \in E\left(H_{i}\right)} f(e)=k n$ because the magic constant is $k$. Since $n$ is even, this is impossible and results in a contradiction to our assumption. Hence, $K_{n, n}-I$ is not an $(n-1)$-star-supermagic decomposable graph.

The necessary condition for the existence of an $(n-1)$ star-supermagic decomposition of $K_{n, n}-I$ is settled directly from Theorem 1 .

Corollary 2. If $K_{n, n}-I$ is $(n-1)$-star-supermagic decomposable, then $n$ is odd or $n \equiv 0(\bmod 4)$.

Next we establish a structural lemma which will be used in the proof of Theorem 4 .

Lemma 3. For any positive integer $m$, the set $[1,12 m]$ can be partitioned into 3 elements subsets which satisfy two conditions as follows:

(i) There are $3 m$ subsets of $[1,12 m]$ such that the summation of all elements in each subset is $18 m+1$.

(ii) There are $m$ subsets of $[1,12 m]$ such that the summation of all elements in each subset is $18 m+3$.

Proof. For $m \in \mathbb{N}$, let $A=[1,4 m]$, let $B=[4 m+1,8 m]$, and let $C=[8 m+1,12 m]$.

Define $f: A \rightarrow B$ by

$$
f(i)= \begin{cases}8 m-2 i+1, & \text { if } i \in[1,2 m], \\ 6 m, & \text { if } i=2 m+1, \\ 12 m-2 i+4, & \text { if } i \in[2 m+2,3 m+1], \\ 12 m-2 i+2, & \text { if } i \in[3 m+2,4 m] .\end{cases}
$$

Let $s_{i}=i+f(i)$ for all $i \in[1,4 m]$. Then the set

$$
\begin{aligned}
S & =\left\{s_{i} \mid i \in[1,4 m]\right\} \\
& =[6 m+1,9 m] \cup[9 m+3,10 m+2] .
\end{aligned}
$$

Now, let $s_{1}^{\prime}=6 m+1$, let $s_{2}^{\prime}=6 m+2, \ldots$, and let $s_{3 m}^{\prime}=9 m$ and let $s_{3 m+1}^{\prime}=9 m+3$, let $s_{3 m+2}^{\prime}=9 m+4, \ldots$, and let $s_{4 m}^{\prime}=$ $10 m+2$. Thus, $S=\left\{s_{i}^{\prime} \mid i \in[1,4 m]\right\}$.

Let $c_{i}=12 m-(i-1)$ for all $i \in[1,4 m]$. Then $C=[8 m+$ $1,12 m]=\left\{c_{i} \mid i \in[1,4 m]\right\}$.

Define $g: S \rightarrow C$ by $g\left(s_{i}^{\prime}\right)=c_{i}$ for all $i \in[1,4 m]$. For each $i \in[1,4 m]$, there exists an element $s_{j} \in S$ such that $s_{j}=s_{i}^{\prime}$, so $g\left(s_{j}\right)=g\left(s_{i}^{\prime}\right)=c_{i}$. Then we have

$$
j+f(j)+c_{i}= \begin{cases}18 m+1, & \text { if } i \in[1,3 m], \\ 18 m+3, & \text { if } i \in[3 m+1,4 m] .\end{cases}
$$

Hence, there are $3 m$ subsets of $[1,12 m]$ such that the summation of all elements in each subset is $18 m+1$, and there are $m$ subsets of $[1,12 m]$ such that the summation of all elements in each subset is $18 m+3$.

Theorem 4. Let $n$ be a positive integer with $n \equiv 0(\bmod 4)$. Then $K_{n, n}-I$ is an $(n-1)$-star-supermagic decomposable graph with the magic constant $\left(2 n^{3}+8 n^{2}-3 n\right) / 4$. 
Proof. Let $n=4 m$ for some positive integer $m$, and let $U=\left\{u_{1}, u_{2}, \ldots, u_{n}\right\}$ and $V=\left\{v_{1}, v_{2}, \ldots, v_{n}\right\}$ be vertexbipartition of $K_{n, n}-I$. Let $\left\{G_{1}, G_{2}, \ldots, G_{n}\right\}$ be an $(n-1)$-stardecomposition of $K_{n, n}-I$, where $G_{i} \cong K_{1, n-1}$ with $V\left(G_{i}\right)=$ $\left\{u_{i}, v_{1}, v_{2}, \ldots, v_{n}\right\} \backslash\left\{v_{i}\right\}$ and $E\left(G_{i}\right)=\left\{u_{i} v_{1}, u_{i} v_{2}, \ldots, u_{i} v_{n}\right\} \backslash$ $\left\{u_{i} v_{i}\right\}$ for all $i \in[1, n]$.

Define a total labeling $f: V\left(K_{n, n}-I\right) \cup E\left(K_{n, n}-I\right) \rightarrow$ $\left[1, n^{2}+n\right]$ by

$$
\begin{aligned}
& f\left(u_{i}\right)= \begin{cases}2 i, & \text { if } i \in[1,3 m], \\
2 i-1, & \text { if } i \in[3 m+1,4 m],\end{cases} \\
& f\left(v_{i}\right)= \begin{cases}2 i-1, & \text { if } i \in[1,3 m], \\
2 i, & \text { if } i \in[3 m+1,4 m],\end{cases}
\end{aligned}
$$

for $i \in[1, n]$, and

$$
\begin{aligned}
& f\left(u_{i} v_{j}\right) \\
& = \begin{cases}2 k n+i, & \text { if } i<j, j=2 k, \\
2 k n+1-i, & \text { if } i<j, j=2 k-1, \\
2 k n+i, & \text { if } i>j, j=2 k-1, j \neq n-3, \\
2 k n+2 n+1-i, & \text { if } i>j, j=2 k, j \neq n-3,\end{cases}
\end{aligned}
$$

for $i, j \in[1, n-3]$, where $i \neq j$.

Note that

$$
\sum_{v \in V\left(G_{i}\right)} f(v)= \begin{cases}n^{2}+\frac{n}{4}+1, & \text { if } i \in[1,3 m], \\ n^{2}+\frac{n}{4}-1, & \text { if } i \in[3 m+1,4 m] .\end{cases}
$$

Now, $n-4$ edges of each $G_{i}$ have been labeled with the partial magic sum $\left(n^{3}-4 n^{2}+n-4\right) / 2$, and their edges are labeled with values from $\left[2 n+1, n^{2}-2 n\right]$. There are still three edges within each $G_{i}$ which need to be labeled, and their label will come from $\left[n^{2}-2 n+1, n^{2}+n\right]$. By applying Lemma 3 to the labeling $f$, we have the following properties:

(i) There are $3 m$ subsets of $\left[n^{2}-2 n+1, n^{2}+n\right]$ such that the summation of all elements in each subset is $3 n^{2}-$ $3 n / 2+1$.

(ii) There are $m$ subsets of $\left[n^{2}-2 n+1, n^{2}+n\right]$ such that the summation of all elements in each subset is $3 n^{2}-$ $3 n / 2+3$.

Thus, $G_{i}$ has the summation of its three remaining edges as $3 n^{2}-3 n / 2+1$ for $i \in[1,3 m]$ and $3 n^{2}-3 n / 2+3$ for $i \epsilon$ $[3 m+1,4 m]$.

Hence, for $i \in[1,3 m]$,

$$
\begin{aligned}
& \sum_{v \in V\left(H_{i}\right)} f(v)+\sum_{e \in E\left(H_{i}\right)} f(e) \\
& =\left(n^{2}+\frac{n}{4}+1\right)+\left(\frac{n^{3}-4 n^{2}+n-4}{2}\right) \\
& \quad+\left(3 n^{2}-\frac{3 n}{2}+1\right)=\frac{2 n^{3}+8 n^{2}-3 n}{4},
\end{aligned}
$$

and, for $i \in[3 m+1,4 m]$,

$$
\begin{aligned}
& \sum_{v \in V\left(H_{i}\right)} f(v)+\sum_{e \in E\left(H_{i}\right)} f(e) \\
& =\left(n^{2}+\frac{n}{4}-1\right)+\left(\frac{n^{3}-4 n^{2}+n-4}{2}\right) \\
& \quad+\left(3 n^{2}-\frac{3 n}{2}+3\right)=\frac{2 n^{3}+8 n^{2}-3 n}{4} .
\end{aligned}
$$

Therefore $K_{n, n}-I$ is an $(n-1)$-star-supermagic decomposable graph, where $n \equiv 2(\bmod 4)$ with the magic constant $\left(2 n^{3}+8 n^{2}-3 n\right) / 4$.

Example 5. Consider the graph $K_{8,8}-I$. Let $U=$ $\left\{u_{1}, u_{2}, \ldots, u_{8}\right\}$ and $V=\left\{v_{1}, v_{2}, \ldots, v_{8}\right\}$ be vertex-bipartition of $K_{8,8}-I$. We have that $K_{8,8}-I$ is 7 -star-supermagic decomposable by using the labeling $f$ in Table 1 with the magic constant 378 .

Theorem 6. Let $n$ be a positive integer, where $n$ is odd and $n \geq$ 3. Then $K_{n, n}-I$ is an $(n-1)$-star-supermagic decomposable graph with the magic constant $\left(n^{3}+5 n^{2}-3 n-1\right) / 2$.

Proof. Let $U=\left\{u_{1}, u_{2}, \ldots, u_{n}\right\}$ and $V=\left\{v_{1}, v_{2}, \ldots, v_{n}\right\}$ be vertex-bipartition of $K_{n, n}-I$. Let $\left\{G_{1}, G_{2}, \ldots, G_{n}\right\}$ be an $(n-1)$-star-decomposition of $K_{n, n}-I$, where $G_{i} \cong$ $K_{1, n-1}$ with $V\left(G_{i}\right)=\left\{u_{i}, v_{1}, v_{2}, \ldots, v_{n}\right\} \backslash\left\{v_{i}\right\}$ and $E\left(G_{i}\right)=$ $\left\{u_{i} v_{1}, u_{i} v_{2}, \ldots, u_{i} v_{n}\right\} \backslash\left\{u_{i} v_{i}\right\}$ for all $i \in[1, n]$.

Define a total labeling $f: V\left(K_{n, n}-I\right) \cup E\left(K_{n, n}-I\right) \rightarrow$ $\left[1, n^{2}+n\right]$ by

$$
\begin{aligned}
& f\left(u_{i}\right)=i, \\
& f\left(v_{i}\right)=n+i,
\end{aligned}
$$

for $i \in[1, n]$, and

$$
f\left(u_{i} v_{j}\right)= \begin{cases}2 k n+i, & \text { if } i<j, j=2 k, \\ 2 k n+1-i, & \text { if } i<j, j=2 k-1, \\ 2 k n+i, & \text { if } i>j, j=2 k-1, \\ 2 k n+2 n+1-i, & \text { if } i>j, j=2 k,\end{cases}
$$

for $i, j \in[1, n]$, where $i \neq j$.

To get the magic constant from the labeling, we compute

$$
\begin{aligned}
& \sum_{v \in V\left(H_{i}\right)} f(v)+\sum_{e \in E\left(H_{i}\right)} f(e) \\
& =\left(f\left(u_{i}\right)+\sum_{\substack{j=1 \\
j \neq i}}^{n} f\left(u_{j}\right)\right)+\sum_{j=1}^{n} f\left(u_{i} v_{j}\right) \\
& =\left(\frac{3 n^{2}-n}{2}\right)+\frac{n^{3}+2 n^{2}-2 n-1}{2} \\
& =\frac{n^{3}+5 n^{2}-3 n-1}{2},
\end{aligned}
$$


TABLE 1: The 7-star-supermagic labeling $f$ of $K_{8,8}-I$.

(a)

\begin{tabular}{lllllllll}
\hline$i$ & 1 & 2 & 3 & 4 & 5 & 6 & 7 \\
\hline$f\left(u_{i}\right)$ & 2 & 4 & 6 & 8 & 10 & 12 & 13 \\
$f\left(v_{i}\right)$ & 1 & 3 & 5 & 7 & 9 & 11 & 14 \\
\hline
\end{tabular}

(b)

\begin{tabular}{|c|c|c|c|c|c|c|c|c|}
\hline$f\left(u_{i} v_{j}\right)$ & $v_{1}$ & $v_{2}$ & $v_{3}$ & $v_{4}$ & $v_{5}$ & $v_{6}$ & $v_{7}$ & $v_{8}$ \\
\hline$u_{1}$ & - & 17 & 32 & 33 & 48 & 52 & 57 & 72 \\
\hline$u_{2}$ & 18 & - & 31 & 34 & 47 & 51 & 59 & 71 \\
\hline$u_{3}$ & 19 & 30 & - & 35 & 46 & 50 & 61 & 70 \\
\hline$u_{4}$ & 20 & 29 & 36 & - & 45 & 49 & 63 & 69 \\
\hline$u_{5}$ & 21 & 28 & 37 & 44 & - & 53 & 60 & 68 \\
\hline$u_{6}$ & 22 & 27 & 38 & 43 & 56 & - & 58 & 67 \\
\hline$u_{7}$ & 23 & 26 & 39 & 42 & 55 & 62 & - & 66 \\
\hline$u_{8}$ & 24 & 25 & 40 & 41 & 54 & 64 & 65 & - \\
\hline
\end{tabular}

for all $i \in[1, n]$. Hence $K_{n, n}-I$ is an $(n-1)$-star-supermagic decomposable graph for all odd $n$.

The following corollary is a direct consequence of Corollary 2 and Theorems 4-6.

Corollary 7. Let $n$ be a positive integer, where $n \geq 2$. Then $K_{n, n}-I$ has an $(n-1)$-star-supermagic decomposition if and only if $n$ is odd or $n \equiv 0(\bmod 4)$.

\section{Conflicts of Interest}

The authors declare that they have no conflicts of interest.

\section{Acknowledgments}

This research was supported by King Mongkut's University of Technology North Bangkok (Contract no. KMUTNB-60GEN-012).

\section{References}

[1] D. B. West, Introduction to Graph Theory, Pretice Hall, New Jersey, NJ, USA, 2001.

[2] A. Gutiérrez and A. Lladó, "Magic coverings," Journal of Combinatorial Mathematics and Combinatorial Computing, vol. 55, pp. 43-56, 2005.

[3] A. Lladó and J. Moragas, "Cycle-magic graphs," Discrete Mathematics, vol. 307, no. 23, pp. 2925-2933, 2007.

[4] A. A. G. Ngurah, A. N. M. Salman, and L. Susilowati, " $H-$ supermagic labelings of graphs," Discrete Mathematics, vol. 310, no. 8, pp. 1293-1300, 2010.

[5] Z. Liang, "Cycle-supermagic decompositions of complete multipartite graphs," Discrete Mathematics, vol. 312, no. 22, pp. 3342-3348, 2012.

[6] G. Marimuthu and S. S. Kumar, "H-V-super magic decomposition of complete bipartite graphs," Electronic Notes in Discrete Mathematics, vol. 48, pp. 231-233, 2015.
[7] J. A. Gallian, "A dynamic survey of graph labeling," The Electronic Journal of Combinatorics, vol. 16, p. S6, 2013.

[8] J. Akiyama and M. Kano, "Path factors of a graph," in Graphs and applications (Boulder, Colo., 1982), Wiley-Intersci. Publ., pp. 1-21, Wiley, New York, 1985. 


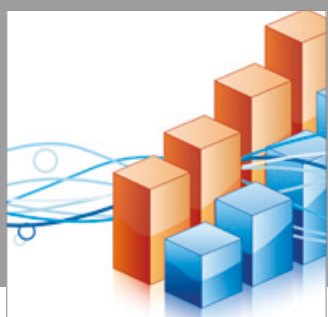

Advances in

Operations Research

vatersals

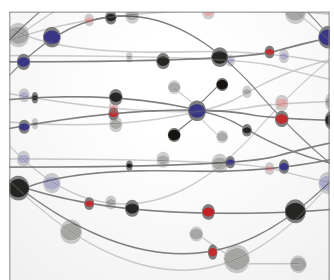

\section{The Scientific} World Journal
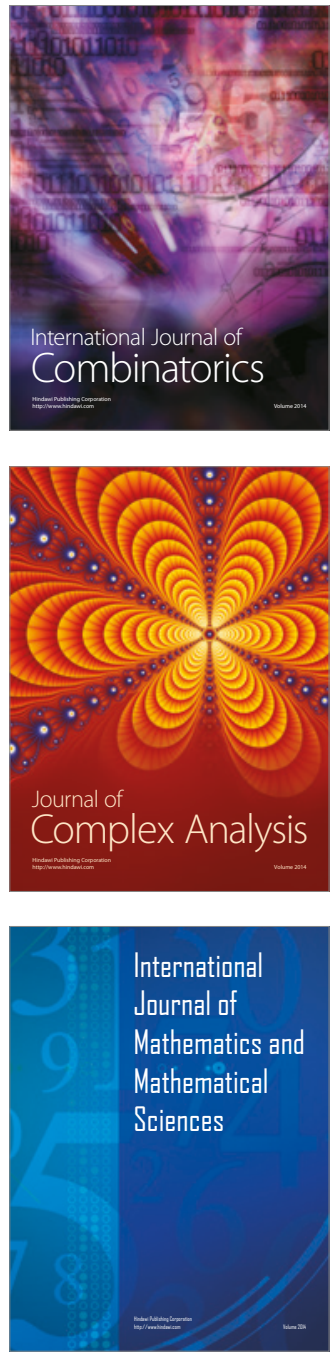
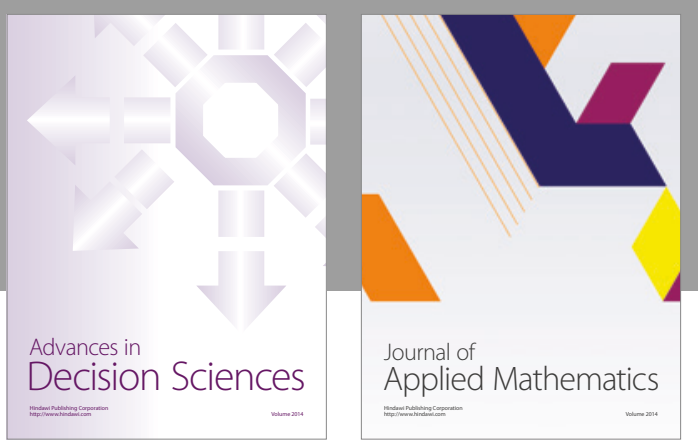

Algebra

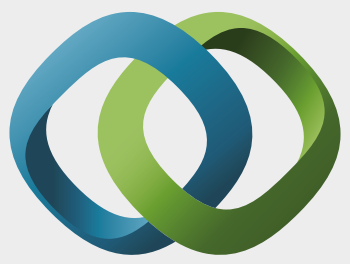

\section{Hindawi}

Submit your manuscripts at

https://www.hindawi.com
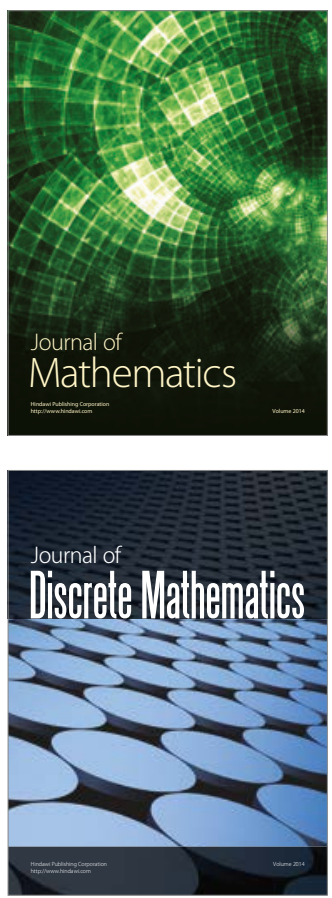

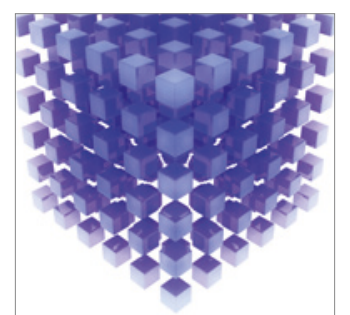

Mathematical Problems in Engineering
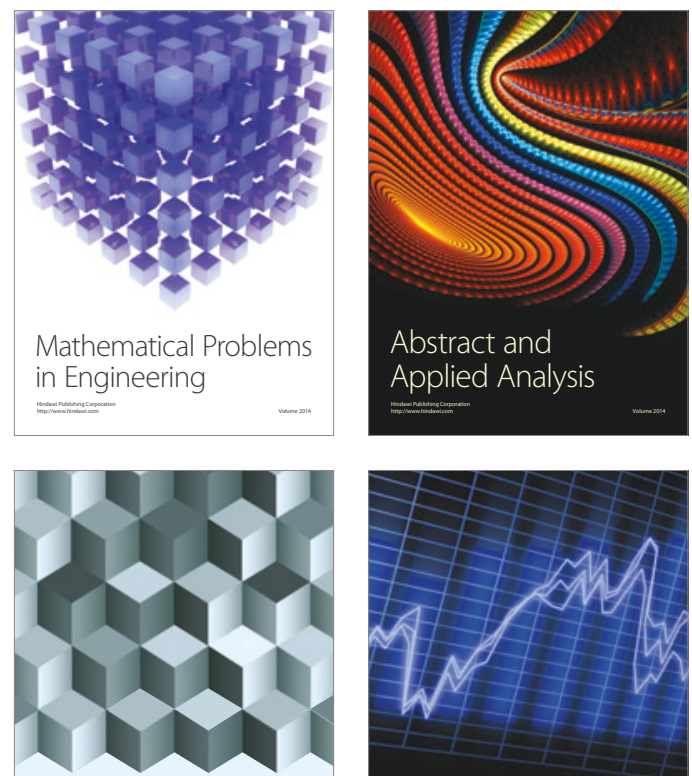

Journal of

Function Spaces

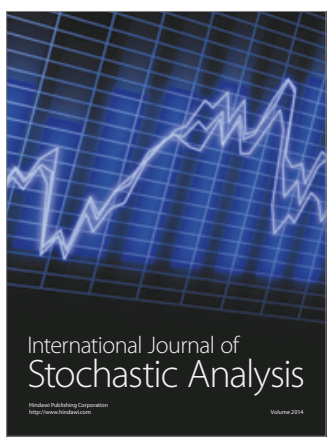

Probability and Statistics
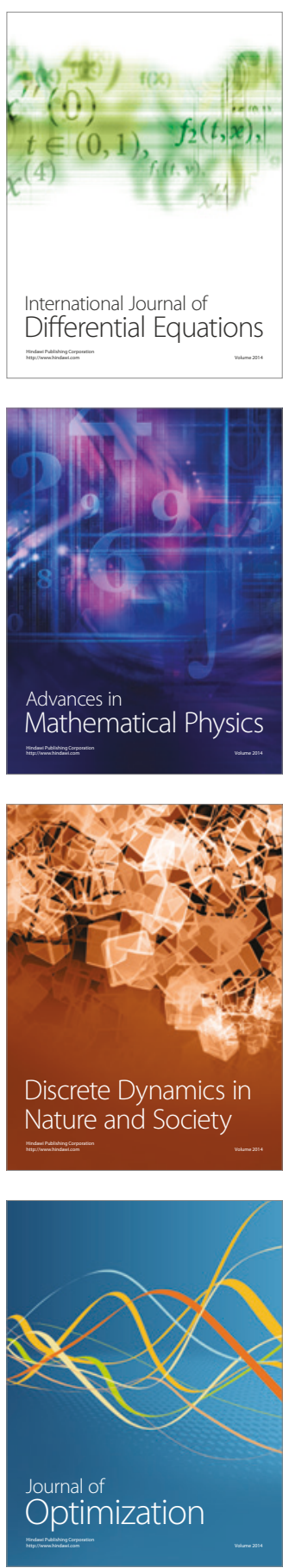